has been made in the general design and construction of transformers in recent years.

\title{
REFERENCES
}

1. Enginering Features of the Boulder Dam-Los ANgeles Lines, 1. F. Scattergood ELEC. ENGG. (A.I.E.E. TRANS.), v. 54, May 1935, p. 494E. F.

2. "Octagonal" Tanks for Large Power Transformers Meet Shipping And Service Requirements, William J. Hart. Steel, v. 97, Sept. 30, 1935, p. 22 .

3. Design features of the World's Largest Transformers, H. L. Cole and F. J. Vogel. Elec. Wld., v. 97, Feb. 21, 1931, p. 374-77.

4 Progress Report on Impulse Testing of Commercial Transformers, F. J. Vogel and V. M. Montsinger. A.I.E.E. Trans., v. 52, June 1933, p. 409-10.

\section{The Sparkless Sphere Gap Voltmeter-II}

\begin{abstract}
Further study of the method of measuring voltages by the force between spheres is reported in this paper, comparison being made of results obtained by means of 100 centimeter spheres with those obtained by other investigators and by a proposed* new A.I.E.E. standard for 50 centimeter spheres.
\end{abstract}

\author{
By \\ R. W. SORENSEN \\ FELLOW A.I.E.E. \\ SIMON RAMO \\ ENROLLED STUDENT A.I.E.E. \\ Both of California Institute of Technology, Pasadena
}

$\mathbf{T}$

HE previous paper ${ }^{1}$ presented the results of certain investigations made to determine the feasibility of using as a measure of voltage the force between a pair of spheres rather than the sparkover distance between them, and compared the results so obtained with spark-over voltages versus spacings for a pair of 100 centimeter spheres mounted along a horizontal axis.

A paper recommended for publication by the A.I.E.E. committee on instruments and measurements, and scheduled for discussion at the A.I.E.E. summer convention, Pasadena, Calif., June 22-26, 1936. Manuscript submitted March 3, 1936; released for publication March 28, 1936.

The authors acknowledge with appreciation the careful and large amount of laboratory work done by Gilbert McCann, Louis Rader, Leonard Patterson, F. V. Maloney, and George Kaneko in obtaining the results used in this paper.

* The A.I.E.E. committee on instruments and measurements appointed a subcommittee in 1933 under the chairmanship of E. J. Rutan to study sphere gap standards and the measurement of impulse voltages. The report of this subcommittee on the sphere gap spark-over voltages for 60 cycles and negative and positive impulses was submitted to the committee for letter ballot, and was approved on April 7, 1936. The recommendation now has been forwarded to the A.I.E.E. standards committee

1. For numbered references see list at end of paper.
Those results were such as to warrant a further study of force between spheres as a means of measuring high voltages. This study as reported here comprises a comparison of voltage as determined by the force on the 100 centimeter horizontally mounted spheres used for the tests described in the previous paper with the spark-over voltage versus spacing for a proposed new A.I.E.E. standard 50 centimeter sphere gap, and a more complete study of force measurements between spheres as a basic or standard method of determining voltage.

In making this study, tests were made on the standard 50 centimeter sphere gap for vertical and horizontal sphere positions at different heights above the floor, the spheres for all tests being mounted in a framework having the recognized proper dimensions.

The following conclusions have been reached as a result of these tests:

1. The sparkless sphere gap voltmeter is worthy of serious consideration as a standard means for measuring high voltages.

2. The proposed new A.I.E.E. standard for the 50 centimeter sphere spark gap is a better calibration than the old standard, but probably indicates for given gap settings voltages which are too low for the larger gap spacings and too high for the smaller spacings.

3. There is no inherent difference between horizontally and vertically mounted sphere spark gaps and the influence of the floor or ground plans is very small for either position when the gaps are of the order of 6 sphere diameters from the ground plane and the gap spacings not over $3 / 4$ the sphere diameter.

4. The sphere spark gap voltmeter is a convenience, but cannot be depended upon as a standard because atmospheric air does not have a constant breakdown voltage, nor does correcting for temperature and barometric pressure account for all variables.

5. The sphere spark gap voltmeter becomes increasingly inconsistent as the gap spacings exceeds $3 / 4$ a sphere diameter.

\section{Conduct of Tests}

Voltage was obtained from the one million volt cascade transformer of the California Institute of Technology. ${ }^{2}$ The effective value of the wave form was 99.25 per cent that of a true sine wave having the same crest value. ${ }^{1}$ Voltage was supplied to the sphere gaps through a one megohm resistance connected as shown in figure 1 .

The dimensions of the laboratory and the arrangement of equipment are shown in figure 1 . The clearances quite evidently are such as to make the influence of building boundaries and equipment on sphere gap performance a reasonable minimum.

A special quick opening circuit breaker was used in the primary of the transformer, thus avoiding the necessity of running water in the resistance used to prevent pitting the spheres on flashover.

The test procedure was as follows: The 50 centimeter sphere gap was set at some desired spacing and a large number of spark-over tests made for each setting, the voltage on the tertiary or voltmeter coil being noted as each flashover occurred. For each test the voltage was started at zero and increased steadily at as rapid a rate as possible consistent with accuracy of tertiary voltmeter reading, the rate of rise being about $20 \mathrm{kv}$ per second. Slower rates of voltage rise are productive of greater variations in spark-over voltage for any given gap setting, the slower rise frequently resulting in flashover at volt- 
ages quite definitely below values specified by A.I.E.E. standards for given gap spacings.

These tests were followed by and interspersed with many tests for determining the relation between tertiary coil voltmeter readings and force measurements on the 100 centimeter sparkless sphere gap for appropriate selected gap spacings and voltmeter settings. Thus the tertiary voltmeter readings were made to serve wholly as comparative readings, making unnecessary a determination of the relation between tertiary voltage and the voltage of the transformer. Readings of transformer voltages as computed from the forces on the spheres were readily reproducible with an accuracy of better than 0.5 per cent for all tertiary voltmeter readings.

The tests were made under varying conditions as to temperature, humidity, and barometric pressure, but all readings have been corrected to $2 \overline{\text { degrees }}$ centigrade and 760 millimeters barometric pressure.

Tests with the 50 centimeter gap were for the most part made with the gap in normal operating position, the axis of the gap vertical and the center of the upper or fixed sphere about 275 centimeters or $5^{1 /} / 2$ diameters above the floor. Two sets of tests were made with the gap horizontal, one at the same height, about $\bar{j}$ diameters above the floor, and the other at a distance of 3 diameters ( 150 centimeters) above the floor.

A fourth set of tests with the gap in a vertical

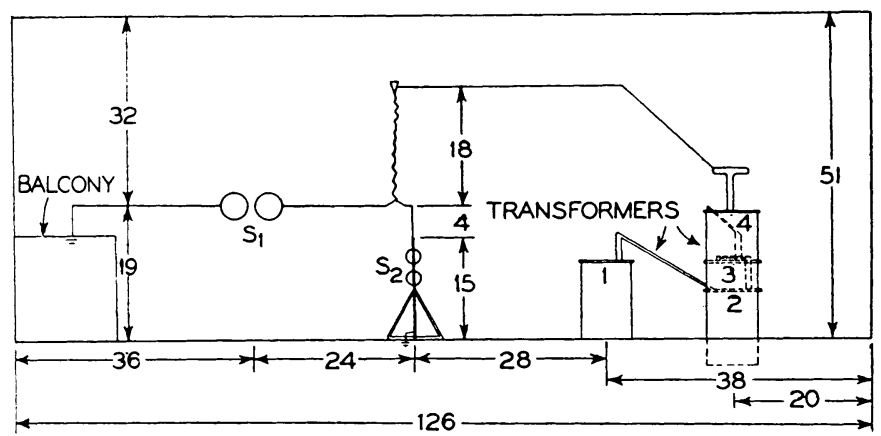

position and over 10 diameters above the floor was also made to check the influence of the floor upon spark-over voltage.

The 2 sets of tests with the gaps in a vertical position gave identical readings for the 2 positions 5 and 10 diameters above the floor for gap spacings up to 50 per cent of the sphere diameter, but for greater spacings there was a definite indication that the higher the gap above the floor the greater the voltage required to produce flashover for given gap settings.

The sets of tests made with the gap horizontal, at standard distance 5 diameters above ground, gave readings which indicate that, with the spheres in this position, correct readings may be obtained for gaps not exceeding half the sphere diameter, but for larger spacings the gap when horizontal flashes over at voltages somewhat lower than for corresponding spacings when the gap is vertical. (See figures 2, 3, and 4.)

With the gap horizontal and only 3 diameters above ground, flashover occurred for all spacings at voltages definitely lower than for like spacings of the gap in its normal operating position. At a spacing of 40 centimeters the voltage in this position was less than 90 per cent the voltage required for flashover in normal position.

In figures 2 and 3 our test points and the results of other investigators are plotted so that the distances of the points from the reference line show the differences between the voltages indicated by the points and the voltages at which, according to a proposed new A.I.E.E. standard, the gap for a given setting should spark over. For each set of tests with the 50 centimeter sphere gap, the gap was conditioned by a number of flashovers before any readings were recorded, but all readings taken after the gaps indicated the ability to give consistent readings, barring, perhaps, a few quite evidently in error, have been plotted. The total spread of points shown for the various gap settings is not the spread found during any one test period, but is that which may be expected for tests extending over several months, and
Fig. 1 (above). Arrangement of apparatus for tests in laboratory

$S_{1}$-Sparkless 100 centimeter spheres $\mathrm{S}_{2}-50$ centimeter spheres in standard frame

Laboratory is 64 feet wide with the test gaps approximately along the center of the building; all dimensions shown are in feet

Fig. 2 (right). Deviation of test points from proposed new standard curves; spheres vertical, lower spheres grounded, upper sphere about $5^{1 / 2}$ diameters from floor

Values obtained by other investigators shown as follows: $X$-Peek ${ }^{3} ; \triangle-$ Bellaschi ${ }^{4} ; \odot-M e a d o r^{5}$

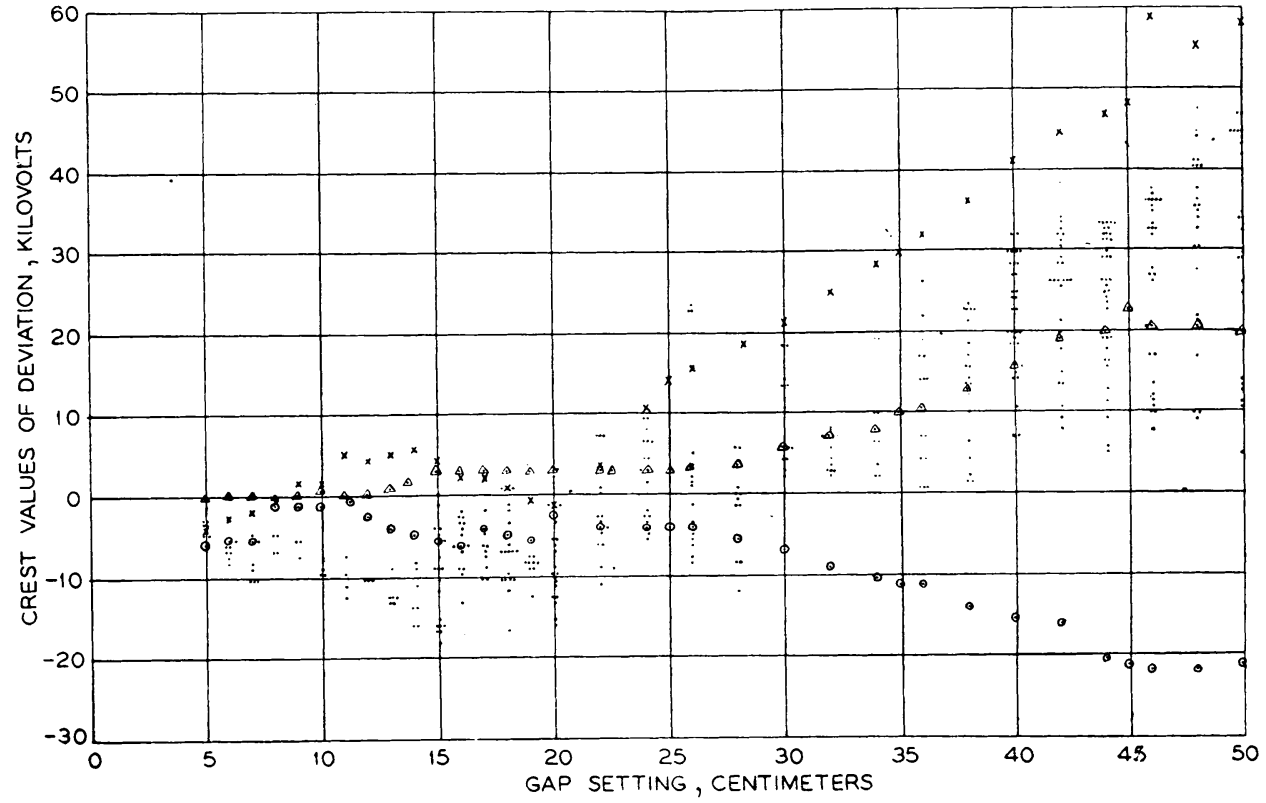




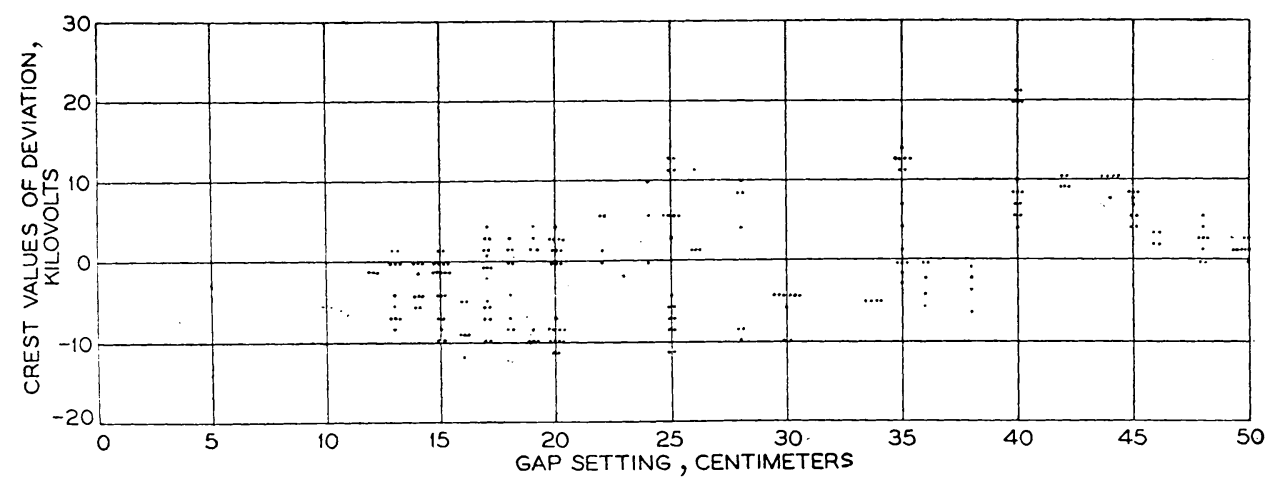

Fig. 3. Deviation of test points from proposed new standard curves for spheres horizontal about $5^{1 / 2}$ diameters above floor

is a spread which apparently cannot be avoided by any amount of care and skill in performing the tests.

The accuracy of voltage determination by means of the sparkless sphere gap is a function of the accuracy used in determining the following:

1. Tertiary voltmeter reading

2. Sphere gap spacings

3. Force

4. Spacing factor $S$ used in the equation $F=S V^{2}$ where $F$ is the force, and $V$ is the voltage impressed on the spheres.

The accuracy of single readings for items 1 to 3 was of the order of 0.2 per cent for tertiary voltmeter readings, $1 / 2$ millimeter for readings of sphere gap settings, and within a gram for force readings, the forces ranging from 100 to over 350 grams.

Mean values of numerous readings made from time to time by different observers and not single readings have been used in determining the relation between tertiary voltmeter readings and the forces between the sparkless spheres at various spacings. The average deviation of single reading values was not more than $0 . \overline{5}$ per cent from a curve plotted through the mean values thus obtained.

The fourth item, spacing factor $S$, can be calculated accurately for isolated spheres. True isolated spheres are, of course, impossible and for actual conditions one must, as in the case of standard sphere gap measurements, take account of supporting shanks and adjacent bodies. The influence of shanks and adjacent bodies on the attractive force between the spheres may be determined in theory by test or by calculation, but in actual operation both methods present difficulties. One test method is that of measuring the capacitances at different spacings for the pair of spheres as a capacitor, and comparing the rates of change of capacitance values with distance to the calculated rates of change for isolated spheres. Such tests call for precise small difference determinations of electrical quantities for apparatus of large physical dimensions. Such determinations are not readily made by methods at our disposal and have not to date yielded satisfactory results.

\section{Determination of Effect of Nearby Objects}

The effect of shanks and nearby objects on the voltage required to exert a force on the spheres can be calculated exactly if enough image points are used. The voltage correction factor curves shown in figures 5 and 6 have been calculated with a sufficient number of image points to assure correction factors having a deviation of only about 5 per cent the absolute correction values which would be obtained with an infinite number of image points. Calculations made by Lord Kelvin, found useful in our image calculations and not readily available, are shown in convenient form in table $\mathrm{I}$. By the use of this table the charge on each sphere and the force between them could be calculated for the condition of neither sphere at ground potential.
Fig. 4. Curves of sparking voltages for 50 centimeter spheres

A-Proposed new A.I.E.E. standard $B$-From Bellaschi

C-Curve through means of points in figure 2

$X$-Means of points in figure 3

$M$-From Meador

P-From Peek

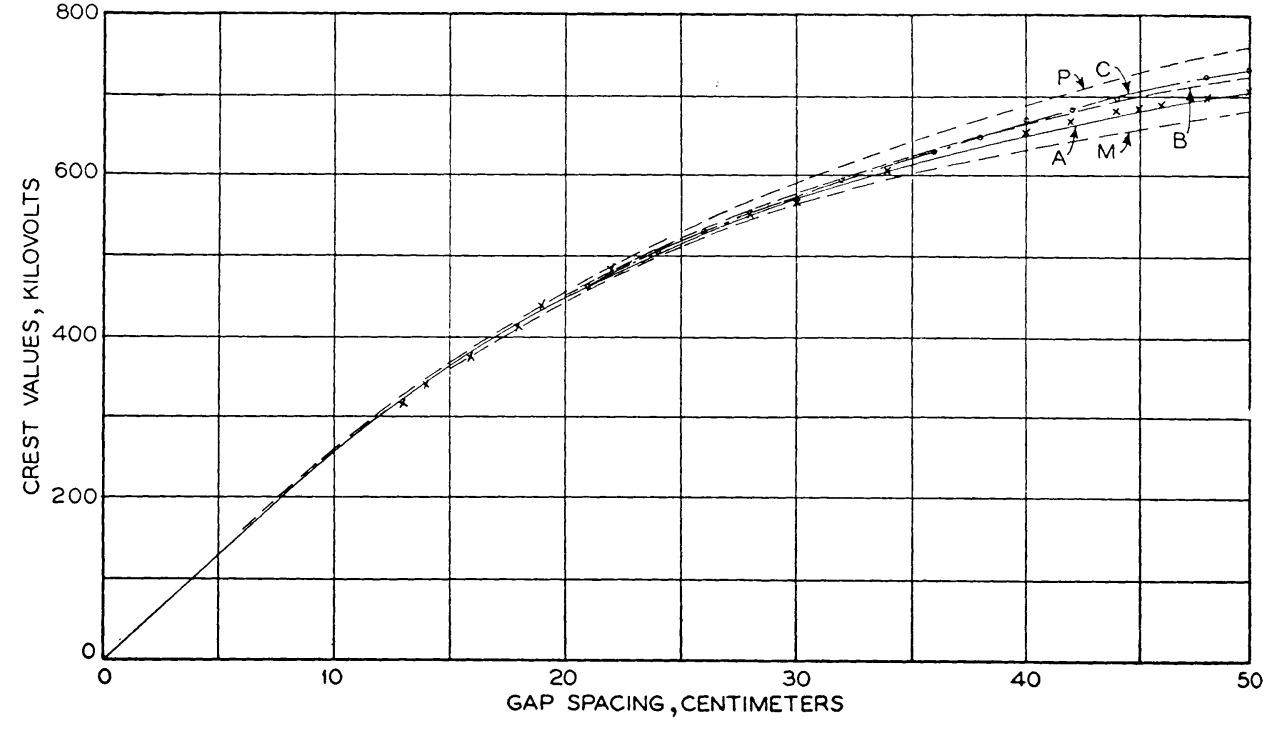

ELECTRICAL ENGINEERING 
Table I-Calculations Made by Lord Kelvin for Sphere Gaps

\begin{tabular}{|c|c|c|c|c|}
\hline $\begin{array}{l}\text { Gap Setting } \\
\text { in Per Cent } \\
\text { of Sphere } \\
\text { Diameter }\end{array}$ & $\frac{\mathrm{C}_{\mathrm{B}}}{\text { radius }}$ & $-\frac{\mathrm{C}_{\mathrm{m}}}{\text { radius }}$ & $\frac{1}{2} \frac{\partial C_{B}}{\partial x}$ & $-\frac{\partial C_{m}}{\partial x}$ \\
\hline $5 \ldots$ & 1.58396 & 0.88175 & 1.13844 & .2 .34878 \\
\hline $10 \ldots$ & 1.43131 . & 0.72378 & 0.52852 . & 1.12700 \\
\hline $15 \ldots$ & 1.34827 & 0.63395 & 0.32917 . & 0.72714 \\
\hline 20 & 1. 29316 & 0.57202 & 0.23159 . & 0.52928 \\
\hline $25 \ldots$ & 1.25324 & 0.52537 & 0.17432 . & 0.41260 \\
\hline $30 \ldots$ & 1. 22218 & 0.48819 . & 0.13696 . & 0.33574 \\
\hline 35 & 1.19755 & 0.45746 & 0.11082 . & 0.28180 \\
\hline 40. & 1.17738 & 0.43140 & 0.09174 & 0.24146 \\
\hline 45 & 1.16056 & 0.40886 & 0.07720 & 0.21052 \\
\hline $50 \ldots$ & 1.14629 . & 0.38908 & 0.06592 . & 0.18598 \\
\hline $55 \ldots$ & 1.13404 & 0.37151 & 0.05693 & 0.16608 \\
\hline 60 & 1.12340 & 0.35571 & 0.04963 & 0.14962 \\
\hline 65 & 1.11410 & 0.34150 & 0.04363 & 0.13582 \\
\hline $70 \ldots$ & 1.10588 & 0.32852 & 0.03863 & 0.12406 \\
\hline 75 & 1. 09859 & 0.31663 & 0.03441 . & 0.11394 \\
\hline $80 \ldots$ & 1. 09208 & 0.30569 & 0.03084 & 0.10514 \\
\hline $85 \ldots$ & 1.08623 & 0.29557 & 0.02775 . & 0.09744 \\
\hline $90 \ldots$ & 1. 08095 & . 0.28617 . & 0.02509 . & 0.09062 \\
\hline 95 & 1. 07617 & 0.27742 . & 0.02278 & .0 .08458 \\
\hline 100 & 1.07182 & 0.26942 . & 0.02075. & .0 .07916 \\
\hline
\end{tabular}

All values are in electrostatic units. The quantities $C_{s}$ and $C_{m}$ are the self- and mutual capacitances, respectively and $\frac{\partial C}{\partial x}$ denotes the rate of change of capacitance with respect to distance between centers. Hence, if the potentials of the spheres are $V_{1}$ and $V_{2}$, then the total charges in each, $Q_{1}$ and $Q_{2}$, and the attractive force are given by:

$Q_{1}=C_{8} V_{1}+C_{m} V_{2} Q_{2}=C_{s} V_{2}+C_{m} V_{1}$

$F=\frac{1}{2} \frac{\partial C_{s}}{\partial x} V_{1}^{2}+\frac{\partial C_{m}}{\partial x} V_{1} V_{2}+\frac{1}{2} \frac{\partial C_{s}}{\partial x} V_{2}^{2}$

The values in table $I$ in combination with the curves of figures 5 and 6 enable calculations to be made for the effect of combinations of planes such as intersecting floor and walls. The effect of these combinations is less than the sum of the influences of the individual planes.

A calculation of the effect of shanks was made for shanks 90 centimeters long and of varying diameter, approximately 5.5 centimeters. This calculation applied to the 100 centimeter spheres for a gap spac-

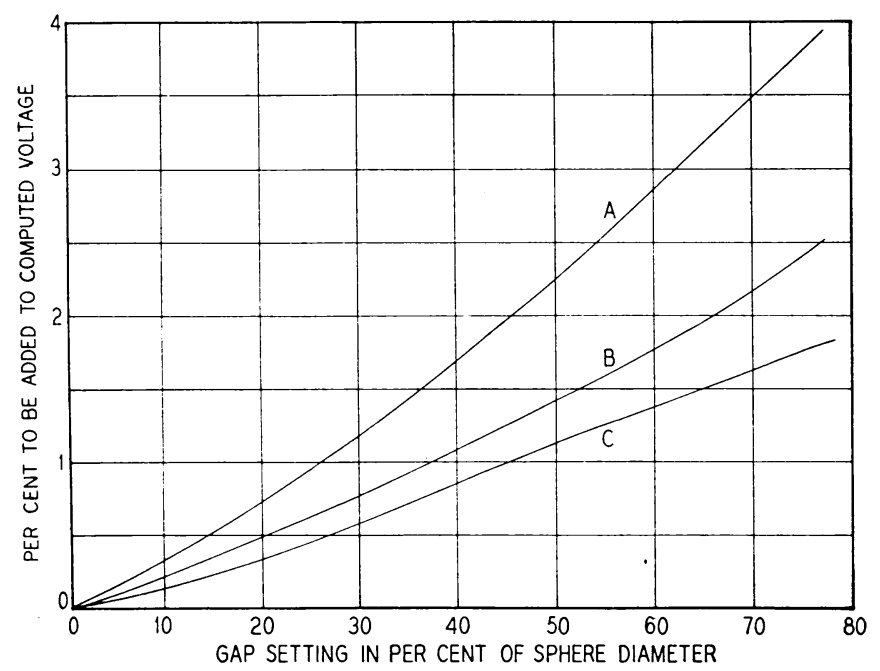

Fig. 5. Curves showing correction for effect of grounded plane parallel to the line of centers of the spheres on the voltage computed from force between spheres

Curves $A, B$, and $C$ for distances above ground of 4,6 , and 8 diameters, respectively

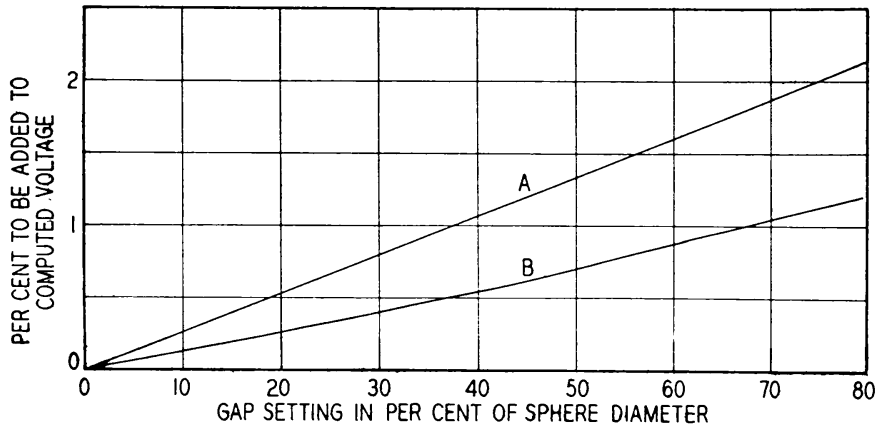

Fig. 6. Curves showing correction for effect of grounded plane perpendicular to the line of centers of the spheres on the voltage computed from force between spheres

Curves $A$ and $B$ for distances of the plane from the spheres of 5 and 10 diameters, respectively; sphere nearest the plane at plane potential

ing of 25 centimeters showed that the voltage as determined by force measurement should be decreased 0.6 per cent. The actual shanks used in the laboratory are $\bar{\jmath} . \overline{5}$ centimeters in diameter and more than 90 centimeters long so the correction should be somewhat more than 0.6 per cent. The influence, however, of increased length is a rapidly decreasing increment.

With the large clearances to floor and walls, and the total influence of the ground planes in a direction opposite that of the shanks and of the same order of magnitude for gap spacings of 25 centimeters or less on the sparkless 100 centimeter sphere gap, it has been considered advisable not to make corrections for these quantities. For greater spacings the influence of ground planes and shanks may not offset each other so nearly. Our large volume of test data for the range of spacing required to compare the readings of the 100 centimeter sparkless gap with the proposed new standard 50 centimeter spark gap shows that at the maximum required setting of the sparkless gap (35 centimeters) the correction for shanks and ground planes would not exceed 1.5 per cent; that is, the voltages indicated by the curves of figures 2 , 3 , and 4 would be decreased by an amount not exceeding this. This small correction, the only one which need be applied for sparkless sphere gaps, and which with further data available may be determined with excellent accuracy, indicates the possibility of using such sphere gaps for reference standards for high voltage measurements.

\section{REFERENCES}

1. The Sparkless Sphere Gap Voltmeter, R. W. Sorensen, J. E. Hobson and Simon Ramo. Elec. ENgG. (A.I.E.E. Trans), v. 54, June 1935, p. 651-6. 2. Development and Characteristics of a 1,000,000-Volt Cascade Transformer at California Institute of Technology, R. W. Sorensen. A.I.E.E. JL., v. 44, Apr. 1925, p. 373-8.

3. Dielectric Phenomena in High Voltage Engineering (a book), F. W. Peek, Jr. McGraw-Hill Book Co., New York, 1929, third edition.

4. Impulse Calibration of Sphere Gaps, P. L. Bellaschi and P. H. Mcauley. Elec. Jl., v. 31, June 1934 , p. 228-32.

5. Calibration of the Sphere Gap, J. R. Meador. Elec. Engg. (A.I.e.e. Trans.), v. 53, June 1934 , p. $942-8$.

6. Discussion, J. R. Meador. Elec. EngG. (A.I.E.E. Trans.), v. 54, Sept 1935, p. 1002-3. 